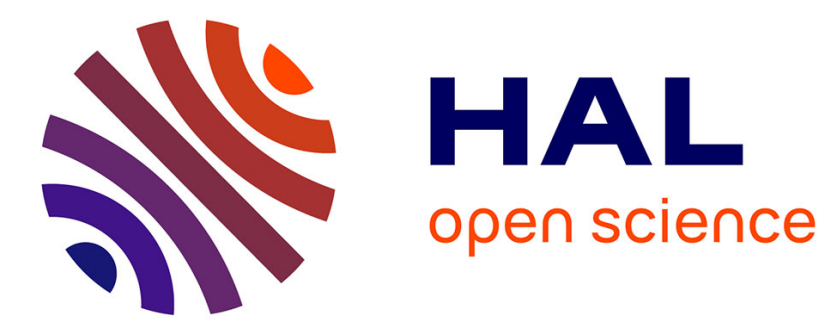

\title{
Experimental evidence of exciton wavefunction shrinkage in InxGa1-xAs/InP multi quantum wells
}

\author{
B. Rotelli, C. Arena, L. Tarricone, C. Rigo
}

\section{To cite this version:}

B. Rotelli, C. Arena, L. Tarricone, C. Rigo. Experimental evidence of exciton wavefunction shrinkage in InxGa1-xAs/InP multi quantum wells. Journal de Physique IV Proceedings, 1993, 03 (C5), pp.C5315-C5-318. 10.1051/.jp4:1993564 . jpa-00251651

\section{HAL Id: jpa-00251651 https://hal.science/jpa-00251651}

Submitted on 1 Jan 1993

HAL is a multi-disciplinary open access archive for the deposit and dissemination of scientific research documents, whether they are published or not. The documents may come from teaching and research institutions in France or abroad, or from public or private research centers.
L'archive ouverte pluridisciplinaire HAL, est destinée au dépôt et à la diffusion de documents scientifiques de niveau recherche, publiés ou non, émanant des établissements d'enseignement et de recherche français ou étrangers, des laboratoires publics ou privés. 


\title{
Experimental evidence of exciton wavefunction shrinkage in $\mathbf{I n}_{x} \mathbf{G a}_{1} x \mathbf{A s} / \mathbf{I n P}$ multi quantum wells
}

\author{
B. ROTELLI, C. ARENA, L. TARRICONE and C. RIGO* \\ Dipartimento di Fisica, viale delle Scienze, 43100 Parma, Italy \\ * CSELT, via G. Reiss Romoli 274, 10184 Torino, Italy
}

\begin{abstract}
We have studied the confinement effect on the ground state of the $n=1$ heavy exciton in $\mathrm{In}_{x} \mathrm{Ga}_{1-x} \mathrm{As} / \mathrm{InP}$ Multi Quantum Wells (MQW) grown by Chemical Beam Epitaxy (CBE). Absorption optical spectra obtained in the excitonic region on several MQW were analyzed as a function of the well thickness $\mathrm{L}_{z}$, ranging in the interval $25-80 \AA$, and as a function of the temperature (8-300 K). In agreement with the theory, a clear enhancement of the oscillator strength calculated by the integrated area of the heavy exciton absorption peak with the well thickness decrease, has been observed; such increase is a direct evidence of the exciton wavefunction shrinkage and is one of the firstly reported in $\operatorname{In}_{x} \mathrm{Ga}_{1-x} \mathrm{As} / \mathrm{InP}$ MQW. The ratio between 1s light and heavy exciton absorption area was used to evaluate their related reduced mass ratio $\mu_{l h} / \mu_{h h}=1.36 \pm 0.075$ in excellent agreement with the calculated one (1.35).
\end{abstract}

\section{INTRODUCTION}

$\mathrm{In}_{x} \mathrm{Ga}_{1-x}$ As lattice matched $(\mathrm{x}=.53)$ to InP appears to be the most promising quantum well (QW) system for realizing new optoelectronic devices; it allows, by varying the well thickness, to tune the detection or emission wavelength within the range $1.3-1.6 \mu \mathrm{m}$, optimum for optical fiber communication systems. The optical absorption of $\mathrm{QW}$ is dominated by exciton peaks which are clearly observable up to room temperature; if an electric field is applied perpendicularly to the QW layers, a red shift and a peak broadening follow according to the well known quantum confined Stark effect. A large variety of applications of this effect (modulators, switches and optical signal processing) has been proposed[1]. In order to achieve an optimum field sensitive exciton absorbtion peak shift a detailed knowledge of the dependence of the exciton properties on the well thickness is highly required. The effect of the reduced dimensionality on the exciton binding energy has been previously studied both theoretically [2-4] and by magnetoabsorption experiments[5]. More recently, by optical absorption measurements performed on AlGaAs/GaAs QW, Masumoto et al. proposed the experimental study of the exciton oscillator strength as a direct probe for investigating the exciton wavefunction properties[6].

In the present work we report an investigation of the oscillator strength of the $(n=1)$ 1s heavy exciton in $\mathrm{In}_{x} \mathrm{Ga}_{1-x} \mathrm{As} / \mathrm{InP} \mathrm{MQW}$ as a function of the well thickness. The results experimentally prove that the oscillator strength has a larger enhancement with the well thickness decreasing in respect to the absorption of the continuum. This is the experimental evidence, at the authors knowledge one of the firstly observed on the $\mathrm{In}_{x} \mathrm{Ga}_{1-x} \mathrm{As} / \mathrm{InP} \mathrm{MQW}$ system, of the two dimensional exciton wavefunction shrinkage. 


\section{EXPERIMENTAL DETAILS}

Unintentionally doped InGaAs/InP Multi Quantum Wells (MQW) were grown at CSELT Labs. by Chemical Beam Epitaxy (CBE) in a VG80H system with an high pressure hydride cell and inlet valves very close to the deposition chamber in order to ensure fast switching of group $V$ species. The metallorganics are injected via in home (CSELT) built pressure controller lines with a specially designed effusion cell for the correct premixing of the reactants. The MQW heterostructures were obtained by alternate growth of $\operatorname{In}_{x} \mathrm{Ga}_{1-x} \mathrm{As}$ and $\operatorname{InP}$ layers on low EPD S-doped InP substrates; all the substrates were back polished to minimize the light scattering. Most of the samples were grown with a little lattice mismatch $(0.17 \%)$ which gives rise to a moderate tensile stress. Asymmetrical growth interruption times were used to minimize the incorporation of the competing hydride and to optimize the well profile[7]. A reference cap of InGaAs with the same composition of the well was utilized to check the ternary composition and removed before measurements by a selective etch. The structure of the QW here investigated is illustrated in Fig.1 and the significant parameters reported in table 1 : here $L_{b}$ and $L_{z}$ are respectively the barrier and the well thickness. Optical transmission measurements were carried out on the samples kept at a controlled temperature into a close cycle Helium cryostat $(8-300 \mathrm{~K})$. Before optical measurements the InP substrate and cladding layers were not removed, thus possible stress to the heterostructure was avoided; in fact, as experimentally proved, InP is transparent in the spectral region of exciton peaks. Transmission spectra were obtained through a halogen lamp illuminating the entrance slit of $0.5 \mathrm{mt}$. focal length Spex monochromator with $\mathrm{PbS}$ photosensor and lock-in detection technique; absorbance measurements were also directly performed by a Varian 2390 spectrophotometer.

TABLE 1

\begin{tabular}{||l|l|l|l|l|l|l|l||}
\hline Sample & qw371 & qw401 & qw412 & qw300 & qw370 & qw135 & qw191 \\
\hline$x \ln$ & 51 & 51 & 51 & 51 & 51 & 52.5 & 51 \\
$(1-x)$ Ga & 49 & 49 & 49 & 49 & 49 & 47.5 & 49 \\
\hline$L_{z}(\AA)$ & 25 & 57 & 57 & 63 & 68 & 76 & 94 \\
$L_{b}(\AA)$ & 385 & 80 & 80 & 100 & 250 & 78 & 80 \\
Periods & 22 & 66 & 66 & 40 & 12 & 60 & 66 \\
\hline
\end{tabular}

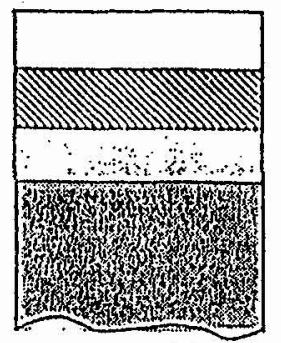

$\operatorname{InP}$

InGaAs/InP MQW

InP

InP substrate: $\mathrm{S}$ doped

Fig.1

Sketch of MQW structure

\section{RESULTS AND DISCUSSION}

Transmission and absorbance data obtained at different temperatures $(8-300 \mathrm{~K})$ in the wavelength region corresponding to the interval between InGaAs and InP energy gap were measured in order to calculate the absorption probability of the MQW. In fact it secms no correct to describe the optical absorption for a MQW system according to the exponential decay law usually used in 3D case. The photon wavelength is much larger than the well width and each photon has a finite probability of being absorbed by the entire quantum well. Consequently it's better to focus the attention on the absorption probability w which is related to the transmittance $T$ by

$$
T=(1-w)^{N}=\exp (N \log (1-w)) \simeq \exp (-N w)
$$


where $N$ is the number of periods[3]. Excitonic peaks ascribed to $n=1$ and $n=2$ interband transitions were observed and the experimental results were compared to the theoretical calculations by founding a fair agreement. Details on the optical measurements and on the exciton energy level calculations which include the strain effect are reported elsewhere[9]. Because the $n=1$ heavy and light exciton peaks remained well defined up to room temperature particularly in the case of the sample qw191, a best fit of the absorption spectra in all the temperature range was attempted by considering two Gaussian functions for the exciton peaks superimposed on a more or less broadened continuum, as described by the relation reported in Ref.[10] and illustrated in Fig.2. In all the other cases a semiempirical fit is obtained by modelling the lineshape of the heavy $(n=1)$ exciton peak through a Gaussian function fitting the low energy side of the absorption peak. The absorption probability shows a plateau above the light exciton peak following the step like character of the two dimensional density of states; its absolute value and the integrated area of the heavy exciton calculated for all the MQW samples are reported as a function of the confinement (i.e. $L_{z}$ value) in Fig.3 and Fig.4 respectively. In Fig. 3 it is possible to see that the dimensionless absorption probability is well width independent. As far as the oscillator strength per unit area $\mathrm{f} / \mathrm{S}$ is concerned, it is related to the absorption probability by

$$
A=\int w(\omega) d(\hbar \omega)=\frac{2 \pi^{2} \hbar e^{2}}{n m_{0} c} \frac{f}{S}
$$

In the quantum well limit the integrated area of the excitonic absorption probability is expressed by the relation $A_{h h} \propto\left|M_{c v}\right|_{h h}^{2} / \lambda^{2}$ where $\lambda$, which represents the average exciton radius along the $x y$ direction (being the $z$ direction perpendicular to the QW layers), decreases with $\mathrm{L}_{z}$. As it is observable in Fig.4, the oscillator strength is expected to enhance rapidly when $L_{z}$ decreases; the experimental data (symbols) have been fitted considering a function $\mathrm{L}_{z}^{-\gamma}$ (continuous line) where $\gamma \simeq 1$ resulted. This fact is a direct experimental evidence of the exciton wavefunction shrinkage in MQW and is in full agreement with similar experiments reported by Masumoto et al.[6] even if these authors have used a different theoretical approach. In the case of qw191 it has been possible to fit both the two excitonic peaks hh1 and $\mathrm{lh} 1$ and calculate the related integrated areas and their ratio in all the investigated temperature range. Within the approximation of weak light intensity, the theory predicts that the integrated area of absorption contains only temperature independent terms; in fact we found that both the $A_{h k}$ and $A_{l h}$ areas and their ratio were temperature independent. By considering[6]:

$$
A_{t h 1} / A_{h h 1}=\left|M_{c v}\right|_{l h}^{2}\left|M_{c v}\right|_{h h}^{2}\left(\mu_{l h}^{2} / \mu_{h h}^{2}\right)=1 / 3\left(\mu_{l h}^{2} / \mu_{h h}^{2}\right)
$$

and

$$
1 / \mu_{h h}=1 / m_{c}+1 / m_{0}\left(\gamma_{1}+\gamma_{2}\right) \quad, \quad 1 / \mu_{h h}=1 / m_{c}+1 / m_{0}\left(\gamma_{1}-\gamma_{2}\right)
$$

where $\gamma_{1}$ and $\gamma_{2}$ are the Luttinger parameters, having assumed the values $m_{c}=.045, \gamma_{1}=13.44$ and $\gamma_{2}=5.37$ corresponding to an In composition $x=.51[11]$, the following values resulted for the reduced masses and for their ratio:

$$
\mu_{l h}=0.033 \quad \mu_{h h}=0.0244 \quad \mu_{h h} / \mu_{h h}=1.35
$$

On the other hand, by assuming the experimental value here obtained for the integrated areas

$$
A_{h h 1}=(1.311 \pm 0.005) 10^{-4} \mathrm{eV} \quad A_{l h 1}=(0.808 \pm 0.04) 10^{-4} \mathrm{eV}
$$

in good agreement with the above calculation the following value resulted:

$$
\mu_{l h} / \mu_{h h}=1.36 \pm 0.075
$$

In conclusion optical absorption experiments are confirmed to be a suitable means to investigate the exciton oscillator strength in MQW, being this latter a direct probe of the exciton wavefunction. In the case of InGaAs/InP MQW we experimentally proved that the absorption probability at the continuum is $\mathrm{L}_{z}$ independent and the integrated area of the heavy exciton increases with $L_{2}$ decrease. At the authors knowledge, this is one of the first experimental evidence of the exciton wavefunction shrinkage reported for the InGaAs/InP MQW and is fully in agreement with previous experiments on AlGaAs/GaAs MQW. Finally the reduced mass ratio of light and heavy excitons has been calculated in satisfying agreement with the theoretical prediction. 


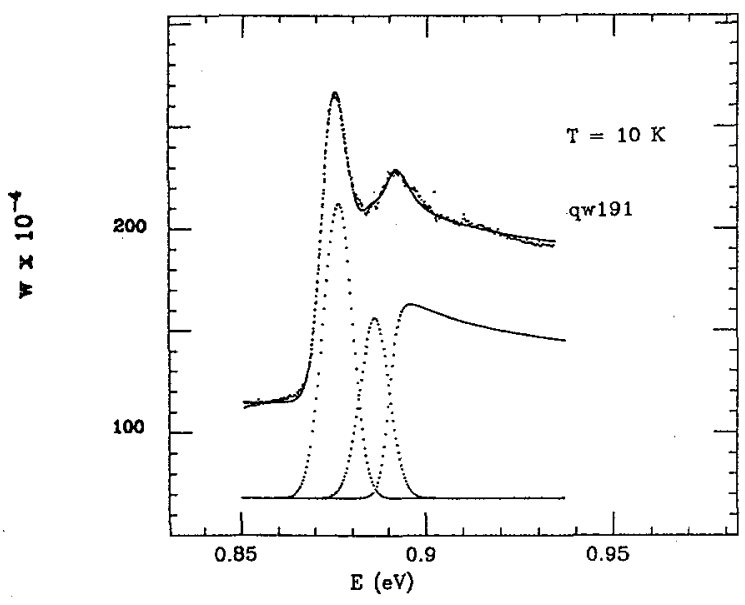

Fig.2

(a) Exciton spectrum of qw191 at $10 \mathrm{~K}$ illustrating the hh1 and $\mathrm{lh} 1$ peaks (dots) and fit result (continuous line). (b)Different contributions to the spectrum represented as two Gaussian functions superimposed to a step-like function.

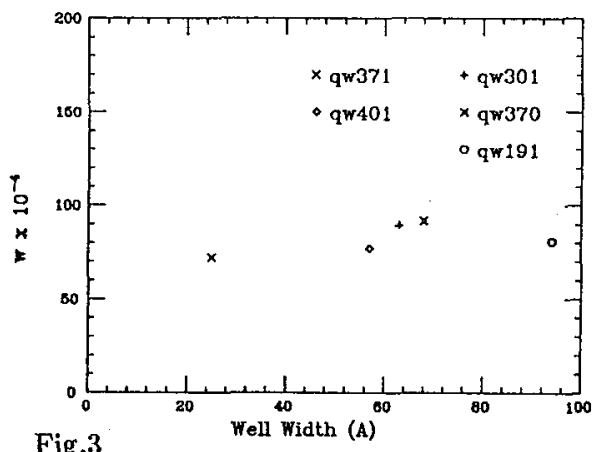

Fig.3

Continuum absorption probability w vs $\mathrm{L}_{z}$ (see table 1)

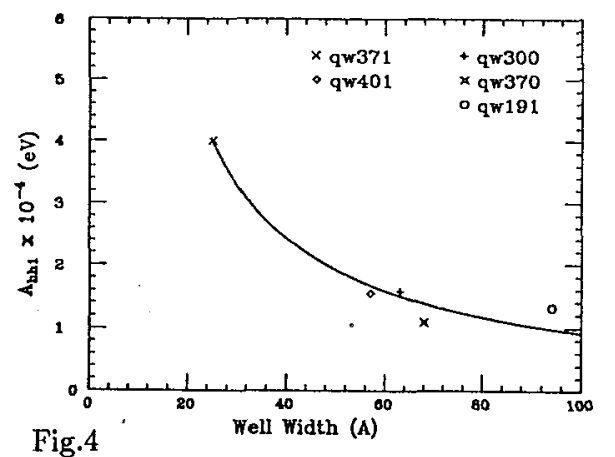

hh1 exciton absorption integrated area vs $\mathrm{L}_{z}$ (see table 1)

\section{ACKNOWLEDGEMENTS}

This work has been undertaken under a joint project CSELT-Dept.Physics of Parma and partially supported by the Progetto Strategico Elettronica dello Stato Solido-CNR. In particular, the authors wish to thank Dr. L.C. Andreani for his helpful discussion and suggestion about a more effective presentation of the absorption data. They are also very grateful to Prof. R. Capelletti for making possible the absorbance measurements and to Dr. F. Genova for his contribution to the CSELT-Physics Dept. of Parma collaboration.

\section{REFERENCES}

[1]T.Y.Wang, K.L.Fry, A.Persson, E.H.Reihlen, G.B.Stringfellow, J. Appl. Phys. 63 (1988) 2674

[2]M.Matsuura, Y.Shinozuta, J. Phys. Soc. Jpn. 53 (1986) 3138

[3]M.Gurioli, J.Martinez-Pastor, M.Colocci, A.Bosacchi, S.Franchi, L.C.Andreani, Phys. Rev. B 47, (1993) 15755

[4]R.L.Greene, K.K. Bajaj, Solid State Commun. 45 (1983) 831

[5]see Ref.7 and Ref.8 in [6]

[6]Y. Masumoto, M.Matsuura, S. Tarucha, H.Okamoto, Phys. Rev. B 32, (1985), 4275; also Surf. Scien. $170,(1986), 635$

[7]G.Salviati, C.Ferrari, L.Lazzarini, F.Genova, C.Rigo, G.M. Schiavini, F.Taiariol, VIII Conference on Microscopy of Semiconducting Materials MSM-VIII Conf.,Oxford 4-8 April 1993, Inst. Phys. Conf. Ser. (to be published)

[8]J.Filipowicz, C.Ghezzi, L.Tarricone, Solid State Commun. 74, (1990), 533

[9]C.Arena, L.Tarricone, F.Genova, C.Rigo, Mat. Sci. and Eng. B (to be published)

[10]D.S.Chemla,D.A.B.Miller, J. Opt. Soc. Am. B 2, (1985), 1155

[11]Landolt-Bornstein, Zahlenwerte und Funktionen aus Naturwissenschaften und Technik, Physik der Elemente der IV Gruppe und der III-V Verbindungen, Springer-Verlag, Berlin 1982 p.223 and p.299. 